\title{
Daño endotelial y activación del sistema hemostático asociado al uso crónico de cocaína: estudios ex vivo e in vitro.
}

\author{
Jaime Pereira, Claudia Sáez, Paulina Olivares, Natalia Moreno, Manuel J Cabrera, \\ Olga Panes, Sabine Belmont, Patricia Hidalgo, Teresa Massardo, Julio Pallavicini, \\ Diego Mezzano. \\ Departamento de Hematología-Oncología, Escuela de Medicina. \\ Pontificia Universidad Católica de Chile y Clínica Siquiátrica Universitaria y Sección Medicina \\ Nuclear, Hospital Clínico Universidad de Chile. \\ Recibido el 23 de Noviembre de 2009, Aceptado el 18 de Febrero de 2010
}

Rev Chil Cardiol 2010; 29: 37-46

\section{Resumen}

Antecedentes: Usuarios crónicos de cocaína tienen riesgo aumentado de presentar infarto de miocardio, angina, muerte súbita y accidentes cerebrovasculares. Aunque la patogenia del daño vascular es mayormente desconocida, se ha encontrado arterioesclerosis prematura y formación de trombos intravasculares.

Objetivo: Demostrar evidencia de daño endotelial y activación del sistema hemostático en usuarios crónicos de cocaína.

Métodos: Un grupo de 23 pacientes con criterios de dependencia a cocaína DSM-IV; 19 hombres (edad promedio 32 a), con exposición a la droga dentro de 72 h del estudio. Disfunción endotelial se evaluó por enumeración de las células endoteliales circulantes (CEC) y nivel de sICAM. Para activación del sistema hemostático se incluyó: complejos trombina-antitrombina (TAT) y generación de trombina; NAP-2 y RANTES para activación plaquetaria. In vitro, CE en cultivo (HUVEC), se expusieron a plasma de consumidores o controles. Se midió factor von Willebrand (FVW) en el medio y expresión de FvW y factor tisular (FT) sobre las CE. Adhesión plaquetaria estática se evaluó por microscopía.

Resultados: En usuarios de cocaína, con respecto a controles, las CEC estaban significativamente elevadas

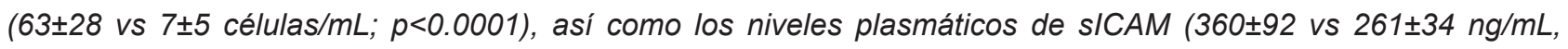
respectivamente; $p<0.01)$. TAT en pacientes y controles fueron $2.7 \pm 0.7$ y $0.7 \pm 0.4 \mu g / L ; p<0.06)$. NAP-2 $(129 \pm 8.4$ $\mathrm{ng} / \mathrm{mL})$ y RANTES $(4.01 \pm 2.12 \mathrm{ng} / \mathrm{mL})$ significativamente aumentados comparados con controles (87.1 \pm 8.6 añd $2.12 \pm 0.35$, respectivamente; $p<0.01)$. El plasma de usuarios de cocaína indujo mayor liberación de FVW desde las HUVEC comparado con plasma control (5.64 \pm 4.0 vs $1.4 \pm 0.8 \mathrm{UI} / \mathrm{dL} ; p \leq 0.001)$. Las HUVEC expuestas a estos mismos plasmas, mostraron aumento significativo en la expresión de FT, FVW y mayor capacidad de adherir plaquetas.

Conclusiones: Consumidores crónicos de cocaína evidencian disfunción endotelial y activación del sistema hemostático. En conjunto con las observaciones in vitro del efecto de los plasmas sobre las CE, estos resultados sugieren que la aterotrombosis puede jugar un papel importante en la patogenia del daño vascular asociado al uso de cocaína.

Trabajo Financiad por Proyecto Fondecyt: 1080253

Correspondencia: Jaime Pereira G.

Banco de Sangre

Hospital Clínico, Pontificia Universidad Católica de Chile

Marcoleta 345

Teléfono: (56-2) 3543385 - Fax: (56-2) 3543772

Correo Electrónico: jpereira@med.puc.cl 


\section{Endothelial damage and activation of the haemostatic system associated to chronic use of cocaine: an ex vivo and in vitro study.}

Background: chronic cocaine users have an increased risk of developing myocardial infarction, angina, sudden death and stroke. Although the pathogenesis of this effect is not completely known, premature atheromatosis and intravascular thrombosis appear to be involved.

Aim: to provide evidence for the presence of endothelial damage and activation of the haemostatic system in chronic cocaine users.

Methods: 23 subjects (19males, overall mean age 32) with DSM-IV criteria for cocaine dependency and exposure to the drug within 72 hours were studied. Endothelial dysfunction was determined by circulating endothelial cell counts (CEC) and sICAM levels. Thrombin-antithrombin complexes (TAT) and thrombin generation were used to characterize haemostatic status. In vitro, platelet activation was studied by NAP-2 and RANTES. EC in culture (HUVEC) were exposed to plasma from cocaine users and controls. Von Willebrand factor was measured in the culture media as well as its expression along with that of tissue factor in EC. Platelet adhesion was evaluated by microscopy.

Results: Compared to controls, EC were significantly increased in cocaine users $(63 \pm 28$ vs. $7 \pm 5$ cells per $\mathrm{mL}$, $p<0.0001)$. Respective s/CAM levels were $360 \pm 92 \mathrm{vs}$. $261 \pm 34 \mathrm{ng} / \mathrm{mL}(p<0.01)$. Similarly, TAT were $2.7 \pm 0.7$ and $0.7 \pm 0.4 \mu \mathrm{g} / \mathrm{L}, \quad(p<0.06)$. Similar findings were obtained for NAP $(129 \pm 8.4 \mathrm{vs}$. $87.1 \pm 8.6 \mathrm{ng} / \mathrm{mL})$ and RANTES $(4.01 \pm 2.12 \mathrm{vs} .2 .12 \pm 0.35 \mathrm{ng} / \mathrm{L})(p<0.01)$. Plasma from cocaine users induced an increased liberation of $v W$ factor from HUVEC compared to control (5.64 $\pm 4.0 \mathrm{vs.} 1.4 \pm 0.8 \mathrm{UI} / \mathrm{dL} ; p \leq 0.001)$. HUVEC exposed to plasma from cocaine users expressed more FT and $v W$ factor and had a greater capacity to induce platelet adhesion.

Conclusion: endothelial dysfunction and activation of the haemostatic system is observed in cocaine users. Along with the observed in vitro effect of plasma from cocaine users upon EC, these results suggest a significant role of athero-thrombosis in the vascular damage associated to cocaine use.

Key Words: Endothelial dysfunction, haemostatic system, cocaine abuse

\section{Introducción}

El consumo de cocaína constituye un problema mayor a nivel mundial, dando origen a morbilidad, mortalidad y costos importantes a los sistemas de salud. En Chile, el consumo de drogas ilícitas se ha estabilizado en los últimos años en alrededor del $6 \%$ de la población entre 12 y 64 años, lo que significa cerca de 475.000 personas (CONACE 2006, Informe Anual de la Situación de las Drogas en Chile; www.conacedrogas.cl/inicio/ pdf/Obser_ok.pdf). De este universo, alrededor del 2\% (135.000 personas) se reconocen consumidores habituales de cocaína $(1.57 \%)$ o pasta base $(0.51 \%)$. Este consumo afecta fundamentalmente a hombres (5:1 con respecto a las mujeres), que tienen entre 19 y 34 años y pertenecen mayoritariamente a los grupos socioeconómicos medio y bajo. Con respecto a la tendencia en el consumo de cocaína, la prevalencia prácticamente se duplica entre el año 1994 y la últimas encuesta del año 2002; sin embargo, desde al año 1998 esta prevalencia se estabilizó en alrededor del $1.5 \%$.

Aunque los efectos sociales y sicológicos del consumo de drogas son ampliamente conocidos, fundamentalmente por la divulgación a través de los medios de comunicación, las consecuencias médicas de este problema son casi desconocidas por los usuarios. De estas, existen complicaciones médicas graves, como por ejemplo, las que afectan el aparato vascular, que pueden derivar en morbilidad y mortalidad significativas ${ }^{1}$. Datos publicados en Estados Unidos y Australia muestran que el uso de cocaína representa la primera causa de muerte relacionada al uso de drogas ilícitas, afectando fundamentalmente a individuos entre 14 y 34 años ${ }^{1,2}$.

Desde el punto de vista clínico, los dependientes de cocaína tienen un riesgo aumentado de presentar 
infarto de miocardio, angina, muerte súbita, y accidentes cerebrovasculares $^{3-12}$, así como defectos regionales de perfusión cerebral (DRPC) $)^{13,14}$. La patogenia de las lesiones vasculares isquémicas asociadas al uso crónico de cocaína no se ha dilucidado completamente y se han descrito varios mecanismos, entre los que destacan: 1) vasoconstricción ${ }^{15-16}$ 2) aumento del consumo de oxígeno y 3) trombogénesis ${ }^{17-18}$.

Diferentes estudios y casos reportados han demostrado claramente un aumento en la formación de trombos arteriales en usuarios de cocaína ${ }^{9}, 18,19 ;$ sin embargo, los estudios in vitro e in vivo sobre sus mecanismos han entregado también resultados controvertidos $^{9,20-22,23-25}$. Minor y cols. ${ }^{9}$ en un estudio en pacientes con infarto agudo del miocardio asociado al uso de cocaína, encontraron que un $38 \%$ presentaba coronarias sanas, pero en alrededor del $80 \%$ había evidencia angiográfica de trombos intracoronarios. El efecto de la cocaína sobre las plaquetas y trombogénesis asociada ha sido estudiado desde hace largo tiempo, pero con una gran heterogeneidad en cuanto a modelos experimentales (animales o humanos), efecto ex vivo o in vitro, exposición aguda o crónica a la droga y diferentes técnicas de laboratorio. En este sentido, es importante señalar que de acuerdo al conocimiento actual sobre el daño vascular aterosclerótico, se ha sugerido que las plaquetas activadas no sólo jugarían un papel en la trombogénesis sino también en la patogenia de la ateroesclerosis ${ }^{26,27}$. Varios estudios han demostrado la capacidad de las plaquetas activadas de inducir una reacción inflamatoria sobre las células de la pared vascular. Cuando las plaquetas se activan expresan CD40L la que al igual que otras citoquinas proinflamatorias, induce activación de las células endoteliales con secreción de quimioquinas tales como la "proteína de quimioatracción para monocitos" (MCP-1) e IL-8 ${ }^{28,29}$, lo que genera señales capaces de reclutar leucocitos y desencadenar una respuesta inflamatoria del endotelio. Por otra parte, la activación de las plaquetas lleva a la secreción de múltiples quimioquinas, incluyendo CCL3, CCL4 (PF4) y RANTES entre otras ${ }^{30}$. Después de su secreción desde las plaquetas, la quimioquina RANTES puede ser inmovilizada sobre la superficie de las células endoteliales. Este depósito de RANTES parece ser muy efectivo en condiciones de flujo para detener plaquetas y subsecuentemente monocitos o células $\mathrm{T}^{31}$. En relación al uso de cocaína, Rinder ${ }^{22}$ encontró aumento de la p-selectina sobre la superficie de las plaquetas en usuarios crónicos de cocaína y el aumento de la p-selectina soluble ha demostrado jugar un papel clave en la generación de un estado procoagulante por acción sobre las células endoteliales $^{32}$ y/o monocitos ${ }^{33}$.

El objetivo de este trabajo fue demostrar, mediante estudios ex vivo e in vitro, que el uso crónico de cocaína se asocia a daño endotelial y activación del sistema hemostático.

\section{Material y Métodos}

Pacientes: Estudiamos 23 dependientes de cocaína (26-53 años, promedio 33) de acuerdo a criterios DSM-IV de la American Psychiatric Association, que ingresaron para tratamiento de desintoxicación a la Clínica Siquiátrica de la Universidad de Chile. Todos los pacientes tenían más de dos años de consumo, con el último dentro de las 72 horas previas al ingreso. En todos ellos se confirmó el consumo reciente por prueba positiva en orina y los que ingresaron al estudio firmaron un consentimiento informado. Se excluyeron aquellos pacientes con patología neurológica, otras co-morbilidades siquiátricas, portadores de patologías asociadas a daño vascular y uso de drogas que afectaran la hemostasia.

Los controles fueron elegidos de una población de individuos aparentemente sanos, con el objetivo de parear de acuerdo a sexo y edad y se estudiaron en forma simultánea con cada paciente.

Estudios ex vivo: Las muestras se tomaron entre 9:00 y 10:00AM en ayunas mediante venopunción atraumática sin uso de ligadura.

La disfunción endotelial se demostró mediante cuantificación de las células endoteliales circulantes (CEC). Brevemente, $1 \mathrm{ml}$ de sangre periférica (EDTA) 
se incubó con $50 \mu l$ de microesferas magnéticas cubiertas con anticuerpo anti-CD146 (Dynabeads). Posteriormente, las microesferas se aislaron mediante un magneto, se lavaron cuatro veces, se marcaron con UEA-1-FITC (Lectina de arbusto ULEX europeo) y se contratiñeron con anti-CD45-PE. Después de incubar 45 min a temperatura ambiente, las microesferas se cargaron en una cámara de Nageotte y se contaron las CEC bajo microscopio de fluorescencia.

Como marcador soluble de disfunción endotelial se determinó los niveles plasmáticos de la forma soluble de la molécula de adhesión intercelular, ICAM (sICAM) por técnica de ELISA (Bender MedSystems, GmbH). Esta misma técnica se usó para demostrar activación de las plaquetas midiendo niveles de NAP-2 y RANTES (Ray Biotech, USA) y CD40L soluble (Bender MedSystems, Germany). La activación del sistema de la coagulación se evaluó mediante determinación de los complejos trombina-antitrombina (TAT) usando ELISA (Affinity Biologicals) y generación de trombina en plasma libre de plaquetas usando la técnica del trombograma calibrado automatizado (Thrombinoscope®, Maastricht The Netherlands).

Estudios in vitro: Las células endoteliales de cordón umbilical (HUVEC) se obtuvieron de acuerdo al método de Jaffe y cols $^{34}$, a partir de cordones umbilicales sin patologías y se cultivaron hasta $90 \%$ de confluencia en multiplacas sobre cubreobjetos cubiertos con gelatina al $1 \%$. Posteriormente las células se cultivaron en medio M199 suplementado con $20 \%$ de plasma libre de plaquetas, obtenido de controles normales 0 de usuarios crónicos de cocaína. En experimentos separados se utilizaron los mismos plasmas, pero previamente depletados de micropartículas mediante filtración. Las células se incubaron por 24 horas, después de lo cual los cubreobjetos se fijaron con etanol y se marcaron con anticuerpos monoclonales específicos para detectar factor von Willebrand (FvW) y factor tisular, mediante microscopía de fluorescencia.

El FVW liberado al medio por las células en cultivo se cuantificó mediante un ensayo de ELISA. Para los estudios de adhesión plaquetaria, las HUVEC expuestas a los plasmas se incubaron con $1 \times 10^{\circ}$ plaquetas por 30 min. Las células se lavaron para eliminar plaquetas no adheridas, se fijaron y se realizó inmuntinciónparalaGPIbdelamembranadelasplaquetas.

Análisis estadístico. Los resultados se expresaron como promedio $\pm \mathrm{DE}$. Para las comparaciones de variables entre pacientes y controles se usó test de "t" de Student o test no paramétrico de Mann-Whitney.

\section{Resultados}

Marcadores de activación del sistema hemostático. Los niveles de NAP-2 (129 $\pm 8.4 \mathrm{ng} / \mathrm{ml})$ y RANTES $(4.01 \pm 2.12 \mathrm{ng} / \mathrm{ml})$ estaban significativamente aumentados comparados con los controles $(87.1 \pm 8.6 \mathrm{ng} / \mathrm{m}$ y $2.1 \pm 0.35 \mathrm{ng} / \mathrm{ml}$, respectivamente; $\mathrm{p}:<0.01$ ), lo que demuestra liberación del contenido de los gránulos alfa (Figura 1).
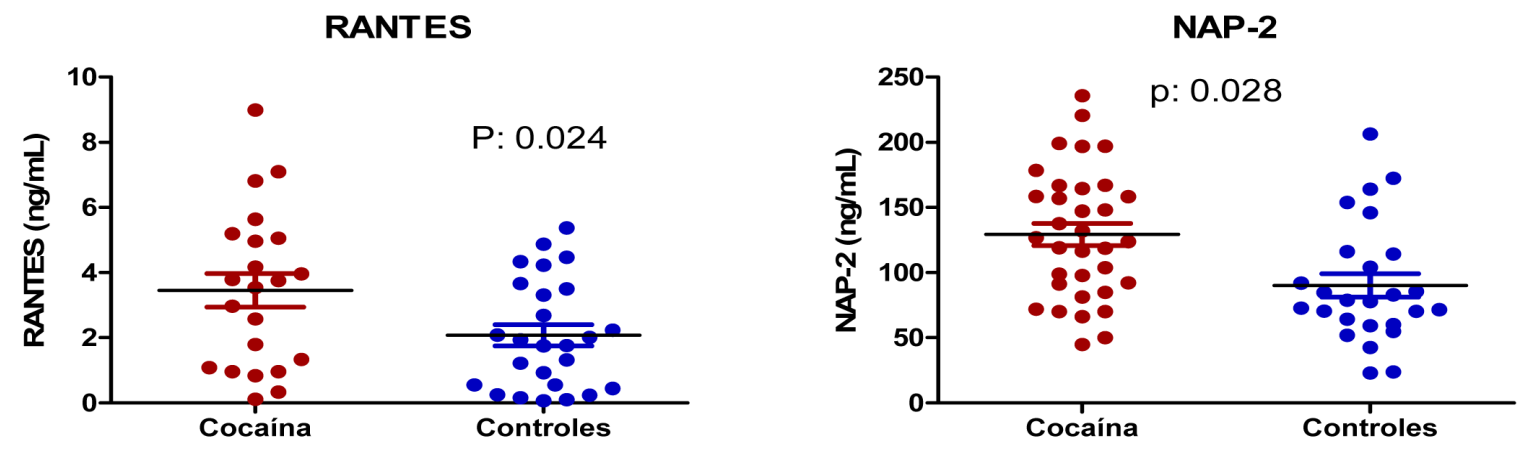

Figura 1: Niveles plasmáticos de las proteínas de gránulos alfa de las plaquetas NAP-2 y RANTES. Las determinaciones se hicieron mediante ensayo de ELISA. 
Otro marcador de activación de las plaquetas, CD40Lsoluble, también se encontró significativamente aumentado en el plasma de los usuarios de cocaína (1.64 \pm 1.0 vs $0.85 \pm 0.5 \mathrm{ng} / \mathrm{mL} ; \mathrm{p}: 0.017)$. Se observó además que los complejos TAT, como marcadores de generación de trombina, se encontraban aumentados con respecto a los controles, aunque este aumento no alcanzó significancia estadística $(2.7 \pm 0.7$ vs $0.7 \pm 0.4$ $\mu g / L ; p: 0.06)$. Sin embargo, la prueba de generación de trombina, que mide la capacidad total del plasma libre de plaquetas de generar trombina estaba significativamente aumentada en los usuarios crónicos de cocaína comparados con los controles. Los dos parámetros que se evalúan con esta técnica, el potencial endógeno de trombina (ETP) y el máximo alcanzado (peak) fueron mayores en pacientes que en los controles (Figura 2).

\section{ETP}

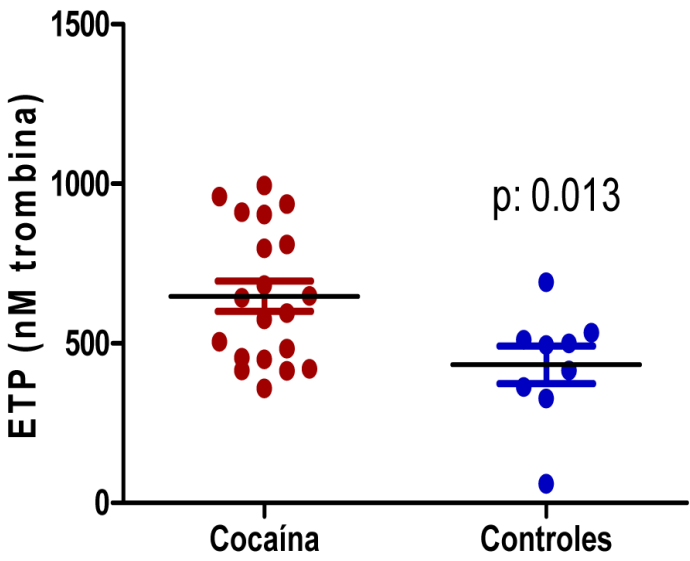

Peak

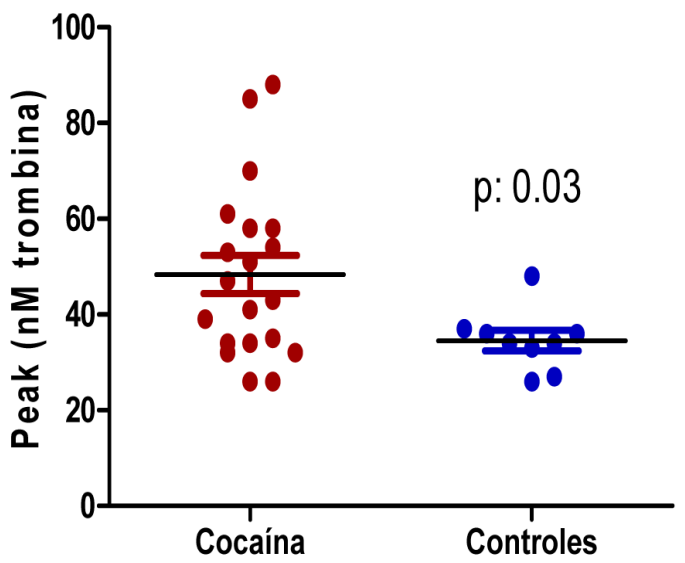

Figura 2. Generación de trombina. La capacidad del plasma para generar trombina se evaluó mediante técnica de trombograma calibrado automatizado, utilizando plasma libre de plaquetas el que fue recalcificado en ausencia de fosfolípidos y factor tisular. En estas condiciones la generación de trombina depende directamente del número de micropartículas presentes en el plasma.

\section{Marcadores de disfunción endotelial}

Los usuarios crónicos de cocaína mostraron un aumento muy significativo en el número de células endoteliales circulantes, encontrándose por sobre el valor de los controles en todos los pacientes (Figura 3).

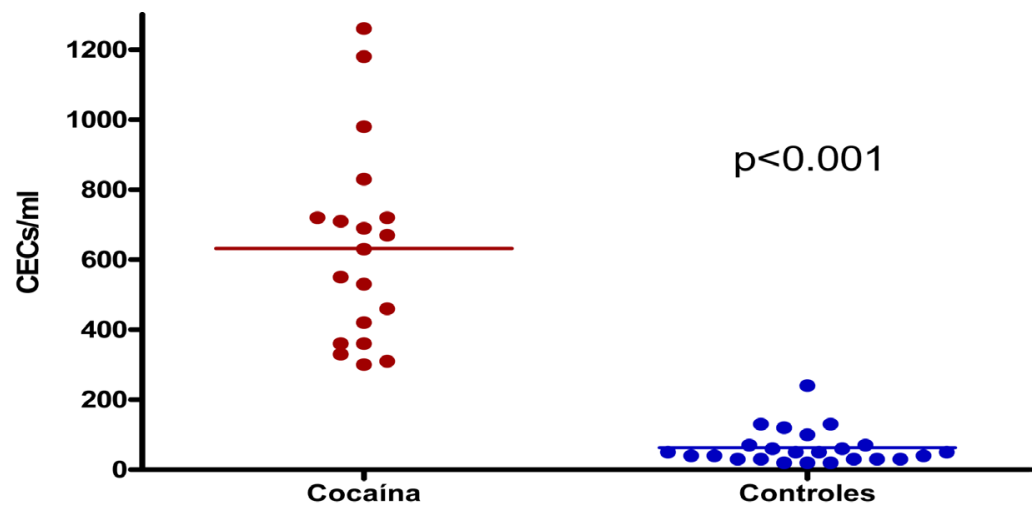

Figura 3. Determinación de las células endoteliales circulantes en sangre periférica. Las células endoteliales se capturaron mediante esferas inmunomagnéticas cubiertas con anti-CD146 y contadas en microscopio de fluorescencia. Se observa un aumento muy importante en el número de células circulantes en los usuarios de cocaína, destacándose que se encuentran elevadas en todos los pacientes estudiados. 


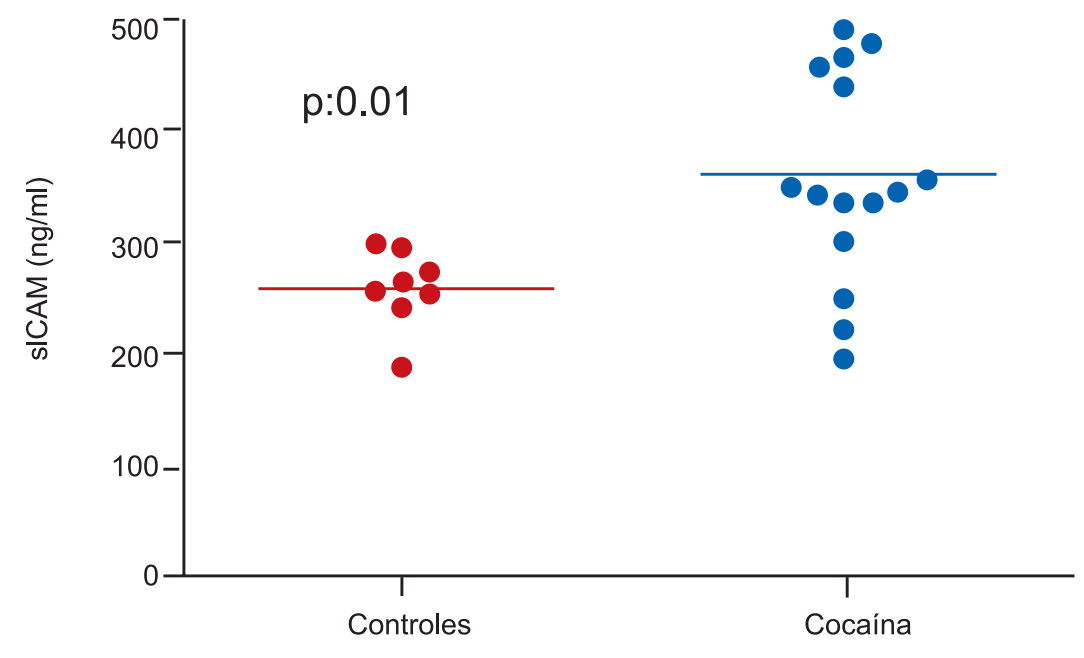

Figura 4. Niveles de ICAM soluble como marcador de daño endotelial. Se observa un aumento significativo del nivel plasmático de esta proteína en los usuarios de cocaína.

\section{Estudios in vitro.}

Las HUVEC expuestas a plasma de los usuarios en la expresión de factor tisular y de FVW sobre la crónicos de cocaína mostraron un aumento significativo membrana (Figura 5).
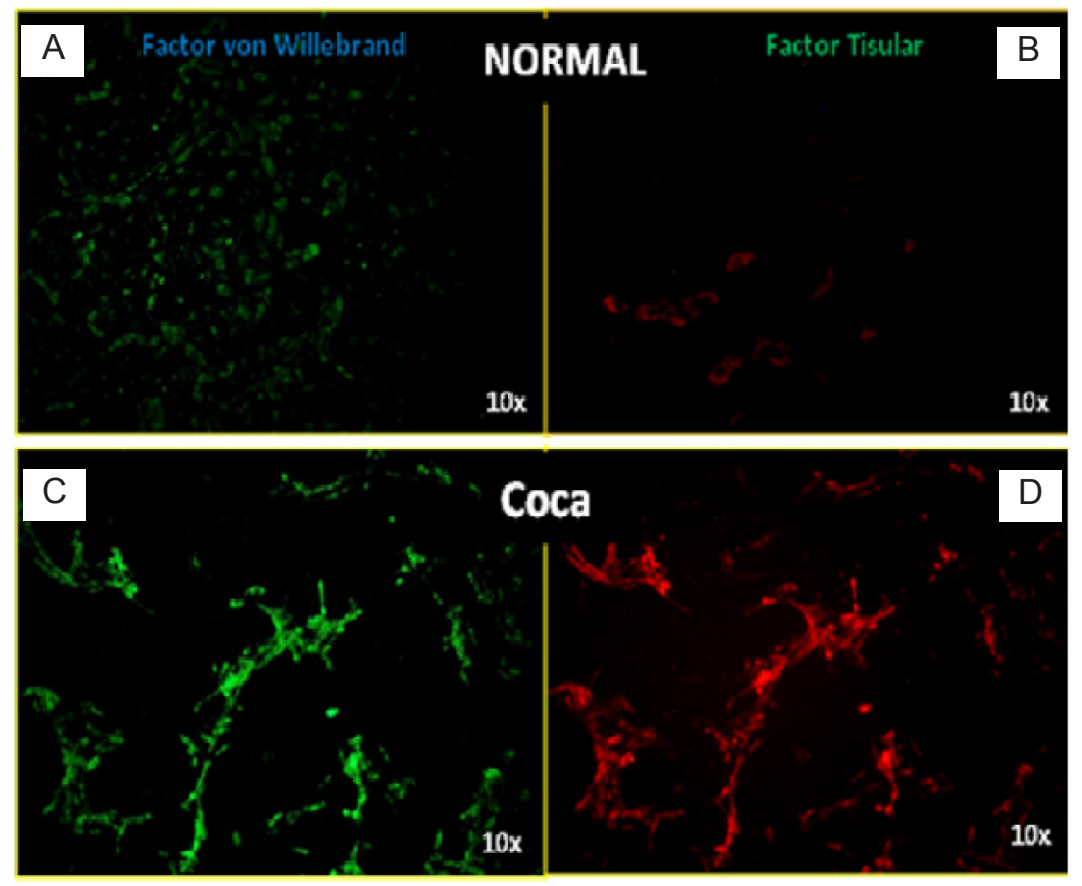

Figura 5. Expresión de factor von Willebrand (FVW) y factor tisular (FT) sobre la superficie de las células endoteliales en cultivo expuestas a plasma normal o de usuarios crónicos de cocaína. En A y B, HUVECs incubadas con plasma normal y teñidas para demostrar FVW y FT, respectivamente. En C y D, las células fueron incubadas con plasma de usuarios de cocaína y teñidas para FVW y FT, respectivamente. Se observa un aumento significativo en la marca tanto para FVW como para factor tisular. 
De igual forma, las HUVEC incubadas con plasma de usuarios de cocaína liberó al medio de cultivo una cantidad significativamente superior de FvW con respecto a los controles, demostrándose además el papel de las micropartículas en este efecto. (Figura 6).

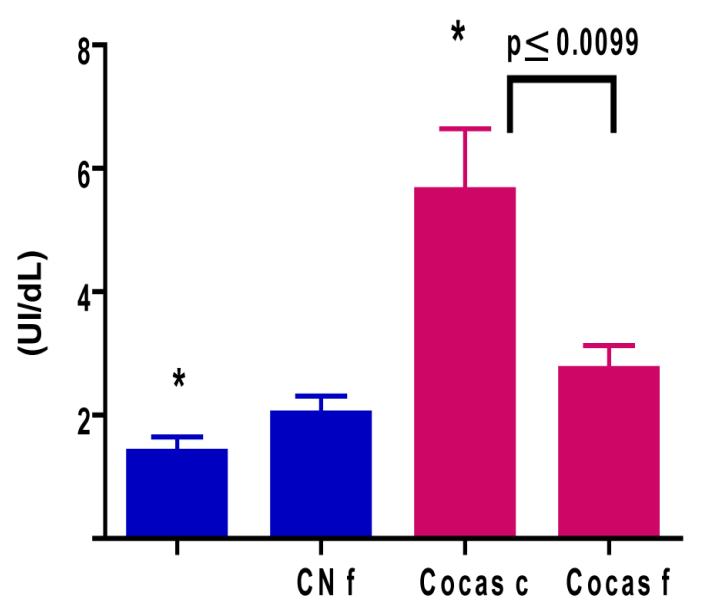

Figura 6. FVW generado por HUVEC. HUVEC suplementadas con plasma de consumidores completo (Coca c) generó mayores niveles de FVW que los controles (CNc) $\left(1,4 \pm 0,8\right.$ vs $5,6 \pm 4,0 \mathrm{Ul} / \mathrm{dL}$; $\left.{ }^{*} p \leq 0,0001\right)$. HUVEC cultivadas con plasma de consumidores depletados de MP (Coca f) induce menor liberación de FvW que el plasma completo (Coca c) (5,6 \pm 4,0 vs 2,7 \pm 1,6 Ul/dL, respectivamente; $p \leq 0,0099)$. Este efecto no se observó en los controles (1,4 $\pm 0,8$ vs 2,0 $\pm 1,0 \mathrm{UI} / \mathrm{dL}$, respectivamente; $p: 0,1532)$. Estos resultados demuestran que el plasma de consumidores de cocaína activa a las células endoteliales y que las MPs contribuyen a la generación de FvW solamente en las HUVEC suplementadas con plasma de consumidores.

En relación a los estudios de adhesión estática de mayor cantidad de plaquetas que las expuestas plaquetas, las HUVEC pre-incubadas con plasma a plasma normal (figura 7). de usuarios crónicos de cocaína adhirieron una
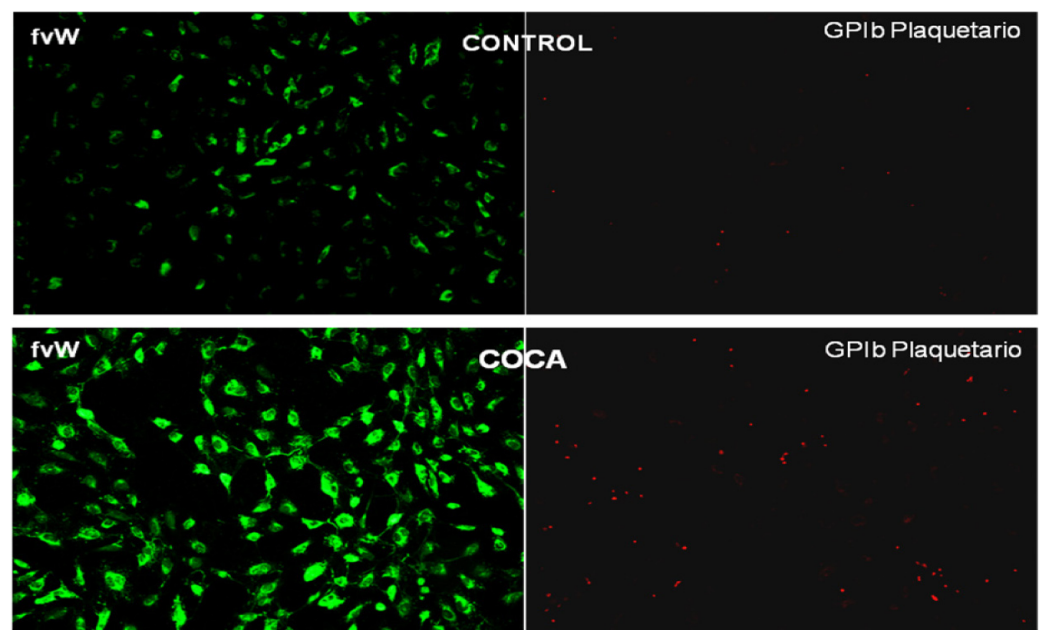

Figura 7. Adhesión de plaquetas a CE en cultivo. HUVEC fueron expuestas a plasma control o de consumidores de cocaína (COCA) (20\%). Después de 24 horas se co-cultivaron con $1 \times 10^{9}$ plaquetas por 30 min. Las células se lavaron para eliminar plaquetas no adheridas, se fijaron y se realizó inmuntinción para la GPlb plaquetaria (rojo) y FvW (verde) (20X)

Consistentemente se observó un mayor número de plaquetas adheridas a las CE expuestas al plasma de consumidores que controles (experimento representativo de 3). 


\section{Discusión}

Los resultados muestran que el uso crónico de cocaína se asocia a una activación del sistema hemostático, evidenciado por marcadores de activación in vivo de las plaquetas y del sistema de la coagulación. Con respecto a la activación plaquetaria, se observó un aumento en los niveles plasmáticos de proteínas propias de los gránulos alfa (NAP-2 y RANTES), lo que denota un proceso de activación con secreción de contenido granular. Cd40L es una proteína que aumenta su expresión sobre la membrana de las plaquetas activadas, pero que es rápidamente escindida y liberada al plasma, constituyendo un muy buen marcador de activación plaquetaria. De hecho, ha sido propuesto como un factor de riesgo de enfermedad cardiovascular ${ }^{35,36}$. En los usuarios de cocaína se encontró niveles significativamente aumentados de CD40L soluble, lo que en conjunto con las observaciones sobre las proteínas granulares, permite sostener que en estos pacientes existe hiperactividad de las plaquetas.

La observación más importante de este estudio creemos la constituye la demostración de daño endotelial, específicamente evidenciado por el importante aumento en el número de células endoteliales circulantes. La disfunción endotelial, definida como la pérdida de las propiedades vasoprotectoras del endotelio, ha sido propuesta como uno de los fenómenos claves en la patogenia de la aterosclerosis ${ }^{37,38}$. La disfunción endotelial se puede evaluar mediante diferentes métodos entre los que se incluyen cambios en el flujo sanguíneo de la arteria braquial (dilatación mediada por flujo, "FMD") ${ }^{39}$ y liberación de marcadores solubles como el $\mathrm{FVW}^{40}$. Recientemente, la medición de las células endoteliales circulantes en la sangre periférica se ha consolidado como la prueba más directa de daño endotelial. Se ha sugerido incluso que pudiera reflejar la intensidad del daño al endotelio, especialmente porque las CECs son muy difíciles de encontrar en la sangre de individuos normales ${ }^{41,42}$. En este estudio encontramos que el número de CECs estaba aumentado en todos los usuarios de cocaína en que se determinaron. Esta evidencia de daño endotelial se confirmó, además, mediante la demostración de niveles elevados de ICAM soluble proteína que aumenta su expresión en la membrana de las células endoteliales activadas/dañadas, desde la cual se escinde y circula en la sangre periférica. Altos niveles de ICAM soluble se han observado en pacientes con aterosclerosis ${ }^{43}$.

Con la intención de ahondar en los mecanismos de daño vascular asociado al uso de cocaína, se realizaron experimentos in vitro para ver el efecto de factores plasmáticos sobre las células endoteliales en cultivo. La exposición de las HUVECs a plasma de usuarios crónicos de cocaína resultó en un aumento en la expresión de FVW y de factor tisular, denotando un cambio de fenotipo de las células hacia uno protrombótico. En esta misma línea se encuentra la observación de que las plaquetas aumentan su adhesión a las células endoteliales que han sido previamente incubadas con plasma de usuarios de cocaína. Este fenómeno de adhesión plaquetaria a las células endoteliales activadas ha sido propuesto como uno de los mecanismos clave en las etapas iniciales del desarrollo de la aterosclerosis ${ }^{44,45}$.

En resumen, el daño endotelial precoz y la activación del sistema hemostático observados, sugieren que los fenómenos de aterotrombosis juegan un papel importante en la patogenia del daño vascular asociado al consumo crónico de cocaína. La persistencia de estas alteraciones podría explicar el desarrollo de aterosclerosis acelerada y eventos isquémicos a edades tempranas.

\section{REFERENCIAS:}

1. LANGE RA, HILLIS LD. Cardiovascular complications of cocaine use. N Engl J Med 2001; 345: 351-358.

2. VASICA G, TENNANT CC. Cocaine use and cardiovascular complications. Med J Aust. 2002; 177: 260-262.

3. CATERALL W, MACKIE K. Local anesthesics, in Hardman
JG, Gilman AG, Limbird LE, (eds). Goodman and Gilman's Pharmacologic basis of therapeutics. (9th ed) New York, NY, McGraw-Hill, 1996, pp 331-338.

4. PITTS WR, LANGE RA, CIGARROA JE, HILLIS LD. Cocaine-induced myocardial ischemia and infarction: 
pathophysiology, recognition and management. Prog Cardiovasc Dis 1997: 40: 65-76.

5. LEVINE SR, BRUST JC, FUTRELL N, BRASS LM, BLAKE $D, F A Y A D P$, et al. A comparative study of the cerebrovascular complications of cocaine: alkaloidal versus hydrochloride: a review. Neurology 1991; 41: 1173-1177.

6. COLEMAN DL, ROSS TF, NAUGHTON JL. Myocardial ischemia and infarction related to recreational cocaine use. West J Med 1982; 136: 444-446.

7. PASTERNACK PF, CALVIN SB, BAUMANN FG. Cocaineinduced angina pectoris and acute myocardial infarction in patients younger than 40 years. Am J Cardiol 1985; 55: 847.

8. ZIMMERMAN FH, GUSTAFSON GM, KEMP HG JR. Recurrent myocardial infarction associated with cocaine abuse in a young man with normal coronary arteries: Evidence for coronary artery spasm culminating in thrombosis. J Am Coll Cardiol 1987; 9: 964-968.

9. MINOR RL JR, SCOTT BD, BROWN DD, WINNIFORD MD. Cocaine-induced myocardial infarction in patients with normal coronary arteries. Ann Intern Med 1991; 115: 797806.

10.HOLLANDER JE, HOFFMAN RS. Cocaine-induced myocardial infarction: an analysis and review of the literature. J Emerg Med 1992; 10: 169-177.

11. HOWARD RE, HUETER DC, DAVIS GJ. Acute myocardial infarction following cocaine abuse in a young woman with normal coronary arteries. JAMA 1985; 254: 95-96.

12. MITTLEMAN MA, MINTZER D, MACLURE M, TOFLER GH, SHERWOOD JB, MULLER JE. Triggering of myocardial infarction by cocaine. Circulation 1999; 99: 2737-2741.

13.MENA I, GIOMBETTI RJ, MILLER BL, GARRETT K, VILLANUEVA-MEYER J, MODY $\mathrm{C}$, et al. Cerebral blood flow changes with acute cocaine intoxication: clinical correlations with SPECT, CT, and MRI. NIDA Res Monogr. 1994; 138:161-73

14.KOSTEN TR, CHEEVES C, PALUMBO J, SEIBYL JP, PRICE LH, WOODS SW. Regional cerebral blood flow during acute and chronic abstinence from combined cocaine-alcohol abuse. Drug Alcohol Depend. 1998; 50: 187-195.

15.LANGE RA, CIGARROA RG, YANCY CW JR, WILLARD JE, POPMA JJ, SILLS MN, et al. Cocaine-induced coronaryartery vasoconstriction. N Engl J Med. 1989; 321:15571562

16.MOLITERNO DJ, WILLARD JE, LANGE RA, NEGUS BH, BOEHRER JD, GLAMANN DB, et al. Coronary-artery vasoconstriction induced by cocaine, cigarette smoking, or both. N Engl J Med. 1994; 17; 330: 454-459.

17.MARTINEZ N, DIEZ-TEJEDOR E, FRANK A. Vasospasm/ thrombus in cerebral ischemia related to cocaine abuse. Stroke. 1996; 27: 147-148

18.KARLSSON G, REHMAN J, KALARIA V, BREALL J. Increased incidence of stent thrombosis in patients with cocaine use. Catheter Cardiovasc Interv 2007; 69: 955958.

19.LEE HO, EISENBERG MJ, DREW D, SCHILLER NB. Intraventricular thrombus after cocaine induced myocardial infarction. Am Heart J 1995; 129: 403405

20.HEESCH CM, WILHELM CR, RISTICH J, ADNANE J, BONTEMPO FA, WAGNER WR. Cocaine activates platelets and increases the formation of circulating platelet containing microaggregates in humans. Heart 2000; 83: 688-695

21.KUGELMASS AD, ODA A, MONAHAN K, CABRAL C, WARE JA. Activation of human platelets by cocaine. Circulation 1993; 88: 876-883

22. RINDER HM, AULT KA, JATLOW PI, KOSTEN TR, SMITH BR. Platelet alphagranule release in cocaine users. Circulation 1994; 90: 1162-1167.

23.TOGNA G, TEMPESTA E, TOGNA AR, DOLCI N, CEBO $\mathrm{B}, \mathrm{CAPRINO} \mathrm{L}$. Platelet responsiveness and biosynthesis of thromboxane and prostacyclin in response to in vitro cocaine treatment. Haemostasis. 1985;15: 100-107

24.KUGELMASS AD, SHANNON RP, YEO EL, WARE JA. Intravenous cocaine induced platelet activation in the conscious dog. Circulation 1995; 91: 1336-1340

25.EICHHORN EJ, DEMIAN SE, ALVAREZ LG. Cocaine induced alterations in prostaglandin production in rabbit aorta. J Am Coll Cardiol 1992; 19: 696-703.

26.WEBER C. Platelets and chemokines in atherosclerosis: partners in a crime. Circ Res 2005; 96: 612-616

27.MASSBERG S, BRAND K, GRÜNER S, PAGE S, MÜLLER $E$, MÜLLER I, et al. A critical role of platelet adhesion in the initiation of atherosclerotic lesion formation. J Exp Med 2002; 196: 887-896

28. HUO Y, SCHOBER A, FORLOW SB, SMITH DF, HYMAN MC, JUNG S, et al. Circulating activated platelets exacerbate atherosclerosis in mice deficient in apolipoprotein E. Nat med 2003; 9: 61-67

29.GAWAZ M, NEUMANN FJ, DICKFELD T, KOCH W, LAUGWITZ KL, ADELSBERGER $\mathrm{H}$, et al. Activated platelets induce monocyte chemotactic protein-1 secretion and surface expression of intercellular adhesion molecule-1 on endothelial cells. Circulation 1998; 98: 1164-1171

30.WAGNER DD, BURGER PC. Platelets in inflammation and thrombosis. Arterioscler Thromb Vasc Biol 2003; 23: 21312137

31.VON HUNDELSHAUSEN $P$, WEBER KS, HUO $Y$, PROUDFOOT AE, NELSON PJ, LEY K, et al. RANTES deposition by platelets triggers monocyte arrest in inflamed and therosclerotic endothelium. Circulation 2001; 103: 17721777

32.ANDRÉ P, HARTWELL D, HRACHOVINOVA I, SAFFARIPOUR S, WAGNER DD. Procoagulant state resulting from high levels of soluble p-selectin in blood. Proc Natl Acad Sci USA. 2000; 97: 13835-13840.

33. CONDE I, NABI F, TONDA R, THIAGARAJAN P, LÓPEZ JA, KLEIMAN NS. Effect of P-selectin on phosphatidylserine exposure and surface-dependent thrombin generation on monocytes. Arterioscler Thromb Vasc Biol 2005; 25: 10651070

34.JAFFE EA, NACHMAN RL, BECKER CG, MINICK CR. Culture of human endothelial cells derived from umbilical veins: identification by morphologic and immunologic criteria. J Clin Invest 1973; 52: 2745-2756.

35. HEESCHEN C, DIMMELER S, HAMM CW, VAN DEN BRAND MJ, BOERSMA E, ZEIHER AM, et al. Soluble CD40 ligand in acute coronary syndromes. $\mathrm{N}$ Engl $\mathrm{J}$ Med 2003; 348: 1104-1111.

36. TANNE D, HAIM M, GOLDBOURT U, BOYKO V, RESHEF T, ADLER Y, et al. CD40 ligand and risk of ischemic stroke or coronary events in patients with chronic heart disease. Int J Cardiol 2006; 107: 322-326. 
J. Pereira, C. Sáez, P. Olivares, N. Moreno, M. J Cabrera, O.Panes, et al.

37.BLANN AD, LIP GY. The endothelium in atherothrombotic disease: assessment of function, mechanisms and clinical implications. Blood Coagul Fibrinol 1998; 9: 297-306.

38. Ross R. Atherosclerosis: an inflammatory disease. N Engl J Med 1999; 340: 115-126.

39.CELERMAJER DS, SORENSEN KE, GOOCH VM, SPIEGELHALTER DJ, MILLER OI, SULLIVAN ID, et al. Non invasive detection of endothelial dysfunction in children and adults in risk of atherosclerosis. Lancet 1992; 340: 11111115.

40.BONEU B, ABBAL M, PLANTE J, BIERME R. Factor-VIII complex and endotelial damage. Lancet 1975; 1: 1430.

41.DIGNAT-GEORGE F, SAMPOL J. Circulating endothelial cells in vascular disorders: new insights into and old concept. Eur J Haematol 2000; 65: 215-220.
42.LEE KW, LIP GYH, TAYEBJEE M, FOSTER W, BLANN AD. Circulating endothelial cells, von Willebrand factor, interleukin-6, and prognosis in patients with acute coronary syndromes. Blood 2005; 105: 526-532.

43.PARKER C, VITA JA, FREEDMAN JE. Soluble adhesion molecules and unstable coronary artery disease. Atherosclerosis 2001; 156: 417-424.

44.GAWAZ M, NEUMANN FJ, DICKFELD T, REININGER A, ADELSBERGER $\mathrm{H}$, GEBHARDT $\mathrm{A}$, et al . Vitronectin receptor (alpha(v)beta3) mediates platelet adhesion to the luminal aspect of endothelial cells: implications for reperfusion in acute myocardial infarction. Circulation 1997; 96: 1809-1818.

45.GAWAZ M. Platelets in the onset of atherosclerosis. Blood Cells Mol Dis 2006; 36: 206-210. 